\title{
Editorial
}

\section{Nonlinear Functional Analysis of Boundary Value Problems 2013}

\author{
Yong Hong Wu, ${ }^{1}$ Lishan Liu, ${ }^{2}$ Benchawan Wiwatanapataphee, ${ }^{3}$ and Shaoyong Lai ${ }^{4}$ \\ ${ }^{1}$ Curtin University, Perth, WA 6845, Australia \\ ${ }^{2}$ Qufu Normal University, Qufu, Shandong 273165, China \\ ${ }^{3}$ Faculty of Science, Mahidol University, Bangkok 10400, Thailand \\ ${ }^{4}$ Southwestern University of Finance and Economics, Chengdu, Sichuan 610074, China \\ Correspondence should be addressed to Benchawan Wiwatanapataphee; benchawan.wiw@mahidol.ac.th
}

Received 6 January 2014; Accepted 6 January 2014; Published 16 February 2014

Copyright (C) 2014 Yong Hong Wu et al. This is an open access article distributed under the Creative Commons Attribution License, which permits unrestricted use, distribution, and reproduction in any medium, provided the original work is properly cited.

Nonlinear boundary value problems play a very important role in the study and control of real world nonlinear systems and the development of new technologies. In recent years, intensive research has been carried out worldwide to develop functional analysis theories and methods for tackling complex boundary value problems arising from scientific research and modelling of real world phenomena. This special issue aims to present some of the recent research developments in this field.

The issue contains twenty seven papers selected through a peer-review process. These papers cover a wide range of topics in nonlinear functional analysis including fixed-point theory, well posedness of nonlinear boundary value problems, asymptotic and stability properties of solutions, derivation of analytical solutions, construction of approximate solutions, and evaluation of the quality of the approximation. The boundary value problems tacked include partial differential equation problems, ordinary differential equation problems with fractional order derivatives and singularities, and stochastic boundary value problems. A brief review of the papers is given below under seven categories.

(1) Well Posedness of Partial Differential Equation Boundary Value Problems

(i) In the paper titled "Nonexistence results for the Schrödinger-Poisson equations with spherical and cylindrical potentials in $R^{3}$," the authors study a Schrödinger-Poisson system leading to the development of two theorems giving two regi- ons on the parameter plane where the system has no nontrivial solutions.

(ii) In the paper titled "The local strong solutions and global weak solutions for a nonlinear equation," the author studies an extended Degasperis-Procesi model and establishes the conditions for the existence and uniqueness of local strong solutions for the underlying nonlinear equation in the Sobolev space.

(iii) In the paper titled "Global existence and uniform energy decay rates for the semilinear parabolic equation with a memory term and mixed boundary condition," the authors establish the global existence and uniqueness of solutions for a mixed initial boundary value problem with a memory term and a generalized Lewis function by the Galerkin method. Estimates for the uniform energy decay rates are also obtained in the paper.

(iv) In the paper titled "Remarks on the blow-up solutions for the critical Gross-Pitaevskii equation," the authors study the blow-up solutions of the critical Gross-Pitaevskii equation for modelling the Bose-Einstein condensate. The sufficient condition for the existence of solutions is established together with some qualitative properties of the minimal blow-up solutions.

(v) In the paper titled "Existence and multiplicity of nontrivial solutions for a class of fourth-order elliptic equations," by using the fountain theorem and the local linking theorem, the authors obtain some existence and multiplicity results for a class of fourth-order elliptic equations. 


\section{(2) Asymptotic and Stability Properties of Solutions}

(i) In the paper titled "On the $L^{1}$ stability to a generalized Degasperis-Procesi equation," by assuming that the strong solutions of the equation are bounded in the sense of $L^{1}(R)$ norm and the initial data is in the space $L^{1}(R) \cap L^{2}(R)$, the authors prove that the solutions are stable in the space $L^{1}(R)$.

(ii) In the paper titled "Asymptotic behavior of global solutions to the Boussinesq equation in multi-dimensions," the Cauchy problem for the Boussinesq equation is investigated. The asymptotic behaviour of the global solutions is established and it is also found that approximation of the global solutions can be obtained by solving the corresponding linear equation as time tends to infinity.

(iii) In the paper titled "Several dynamic properties of solutions to a generalized Camassa-Holm equation," the author investigates some dynamic properties of strong solutions for a generalized Camassa-Holm equation. The results obtained in this work include a one sided upper bound estimate on the first order derivatives of the solution, a space-time highernorm estimate and the $L^{p}(2 p<1)$ bound estimate.

(iv) In the paper titled "Nonlinear instability for a LeslieGower predator-prey model with cross diffusion," the authors investigate the early-stage spatial and temporal patterns formation through a Leslie-Gower predator-prey model with cross diffusion. For any given perturbation near an unstable constant equilibrium, the authors prove that the nonlinear evolution is dominated by the corresponding linear dynamics along a finite number of fastest growing modes.

(v) In the paper titled "Pullback D-attractor of nonautonomous three-component reversible Gray-Scott system on unbounded domains," the author investigates the long time behavior of solutions for the nonautonomous three-dimensional Gray-Scott system with external forcing terms unbounded in the phase space. The existence of a pullback global attractor for the equation is established, and the pullback asymptotic compactness of solutions is proved by using uniform estimates on the tails of solutions on unbounded domains.

\section{(3) Exact and Approximate Solutions of Partial Differential Equation Boundary Value Problems}

(i) In the paper titled "Study of a Newtonian fluid through circular channels with slip boundary taking into account electrokinetic effect," the authors study the slip flow of fluids driving by the combined effect of electrical force and pressure gradient, and the underlying boundary value problem is solved through use of Fourier series expansion in time and Bessel function in space. The results obtained open the way for optimizing the flow by choosing the slip length, the electrical field, and electrolyte solutions.

(ii) In the paper titled "The investigation of solutions to the coupled Schrödinger-Boussinesq equations," by using the expansion method and the symbolic computation system Mathematica, various solutions in terms of hyperbolic functions and trigonometric functions as well as rational functions are obtained for the coupled Schrödinger-Boussinesq equations. The decaying properties of the solutions are also analysed in the paper.

(iii) In the paper titled "Travelling wave solutions for nonlinear Schrödinger equation with a higher-order dispersive term," the author obtains various exact travelling wave solutions for the nonlinear Schrödinger equation with a higherorder dispersive term using the auxiliary equation method, the first integral method, and the direct integral method. The solutions are expressed by Jacobi elliptic functions, hyperbolic functions, and trigonometric functions.

(iv) In the paper titled "The study of the solution to a generalized $K d V-m K d V$ equation," based on an auxiliary equation and the symbolic computation system MATLAB, the authors obtain a number of new exact travelling wave solutions to a generalized $K d V-m K d V$ equation with high order nonlinear terms.

\section{(4) Fractional Order Boundary Value Problems}

(i) In the paper titled "Unique solution of a coupled fractional differential system involving integral boundary conditions," by using the fixed-point theorem for weakly contractive mappings in partially ordered sets, the authors establish the conditions for the existence and uniqueness of positive solutions for a system of fractional differential equations with nonlocal Riemann-Stieltjes boundary integral conditions.

(ii) In the paper titled "Positive solutions for boundary value problems of singular fractional differential equations," by using a fixed-point theorem, the authors establish the existence of positive solutions to a singular fractional boundary value problem involving Caputo fractional derivatives and singularities in the source function.

(iii) In the paper titled "Remark on existence and uniqueness of solutions for a coupled system of multiterm nonlinear fractional differential equations," the authors establish conditions for the existence and uniqueness of solutions for a coupled system of multiterm nonlinear fractional differential equations.

(iv) In the paper titled " $A$ sum operator method for the existence and uniqueness of positive solutions to a system of nonlinear fractional integral equations," based on the fixed-point theorem for the sum operator, the conditions for the existence and uniqueness of positive solutions are established for a Volterra nonlinear fractional system of integral equations. Moreover, an iterative scheme is constructed for approximating the solution.

\section{(5) Singular, Switched Delay, and Sign-Changing Boundary Value Problems}

(i) In the paper titled "Uniqueness and existence of positive solutions for singular differential systems with coupled integral boundary value problems," by the mixed monotone method, the authors establish the sufficient conditions for the existence and uniqueness of positive solutions of a singular differential system with integral boundary condition.

(ii) In the paper titled "Quasilinearization for the boundary value problem of second order singular differential system," the authors extend the existing quasilinearization method to 
solve a class of boundary value problems for second-order singular differential equations.

(iii) In the paper titled "Existence and uniqueness of solution to nonlinear boundary value problems with sign-changing Green's function," by using the cone theory and the Banach contraction mapping principle, some existence and uniqueness results are established for a nonlinear higher-order differential equation boundary value problem with signchanging Green's function.

(iv) In the paper titled "On boundedness and attractiveness of nonlinear switched delay systems," the authors study the boundedness and attractiveness problems for a class of nonlinear switched delay systems. Some sufficient conditions are established to guarantee the system's boundedness. The authors also work out the region where the solution will remain and the relationship between the initial function and the bounded region.

(v) In the paper titled "Unbounded solutions of asymmetric oscillator," the authors establish sufficient conditions for the existence of unbounded solutions of a nonlinear differential equation with a continuous, bounded, and periodic source function.

\section{(6) Stochastic Differential Equation Models}

(i) In the paper titled "A stochastic string with a compound poisson process," the authors introduce a compound Poisson process with a constant jump intensity and random jump-size to capture information burst and the resulting discontinuous path and derive the no-arbitrage condition on the drift of instantaneous forward rates in the compound model. With the proposed model, the impact of random jump on the price of the zero coupon bond is shown in the paper.

(ii) In the paper titled "Equilibrium asset and option pricing under jump diffusion model with stochastic volatility," the authors study the equity premium and option pricing under a jump-diffusion model with stochastic volatility. The pricing kernel is established and the exact expression for the option price is derived by using the Fourier transformation method.

\section{(7) Fixed-Point Theory}

(i) In the paper titled "Generalizations of fixed-point theorems of Altman and Rothe types," the authors present some extensions of the Altman and Rothe type fixed-point theorems. Some new fixed-point theorems for continuous operators are established by use of the theory of topological degree.

(ii) In the paper titled "Common fixed-points for weak contractive mappings in ordered metric space with applications," the authors establish some new common fixed-point theorems satisfying a weak contractive condition in the framework of partially ordered metric spaces.

Yong Hong Wu

Lishan Liu

Benchawan Wiwatanapataphee

Shaoyong Lai 


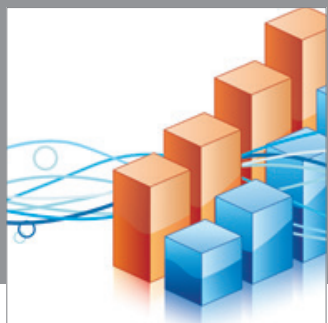

Advances in

Operations Research

mansans

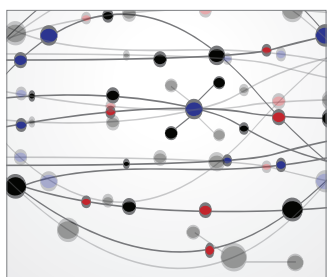

The Scientific World Journal
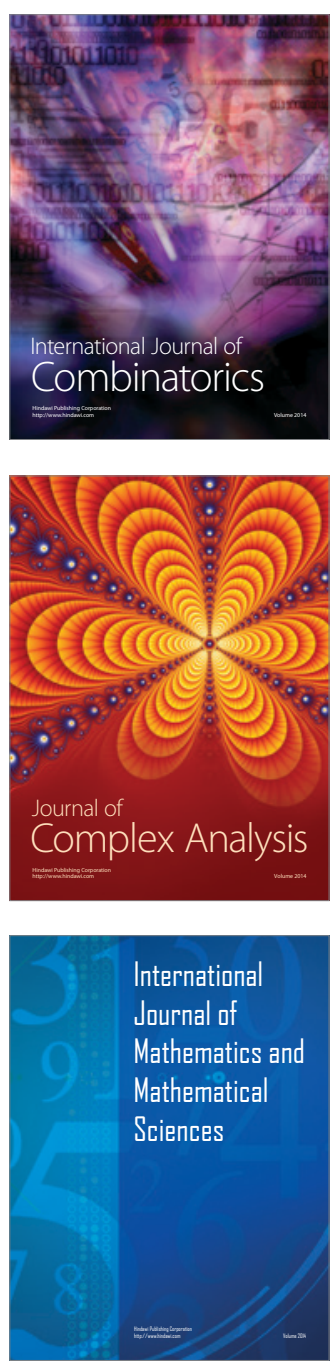
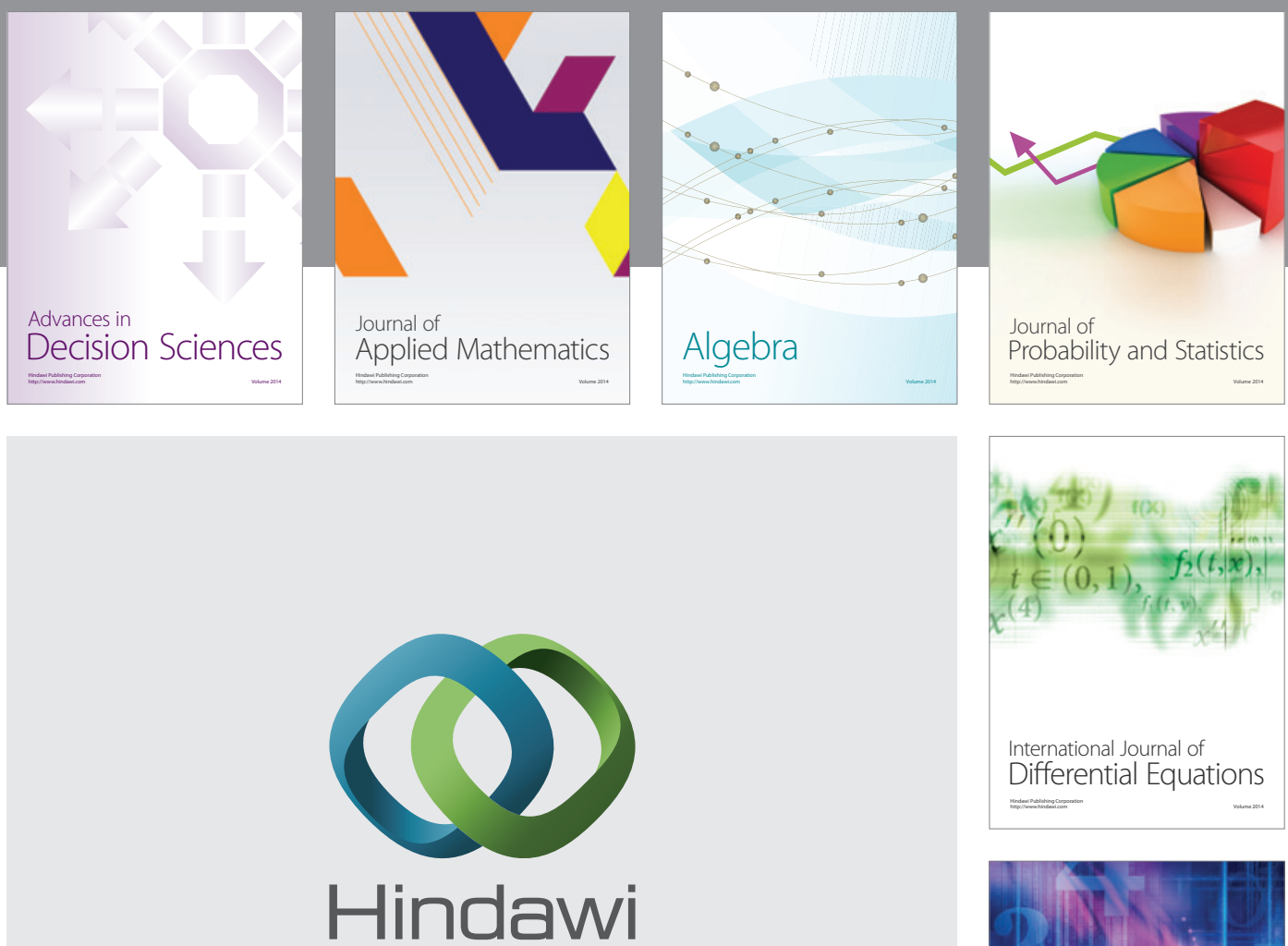

Submit your manuscripts at http://www.hindawi.com
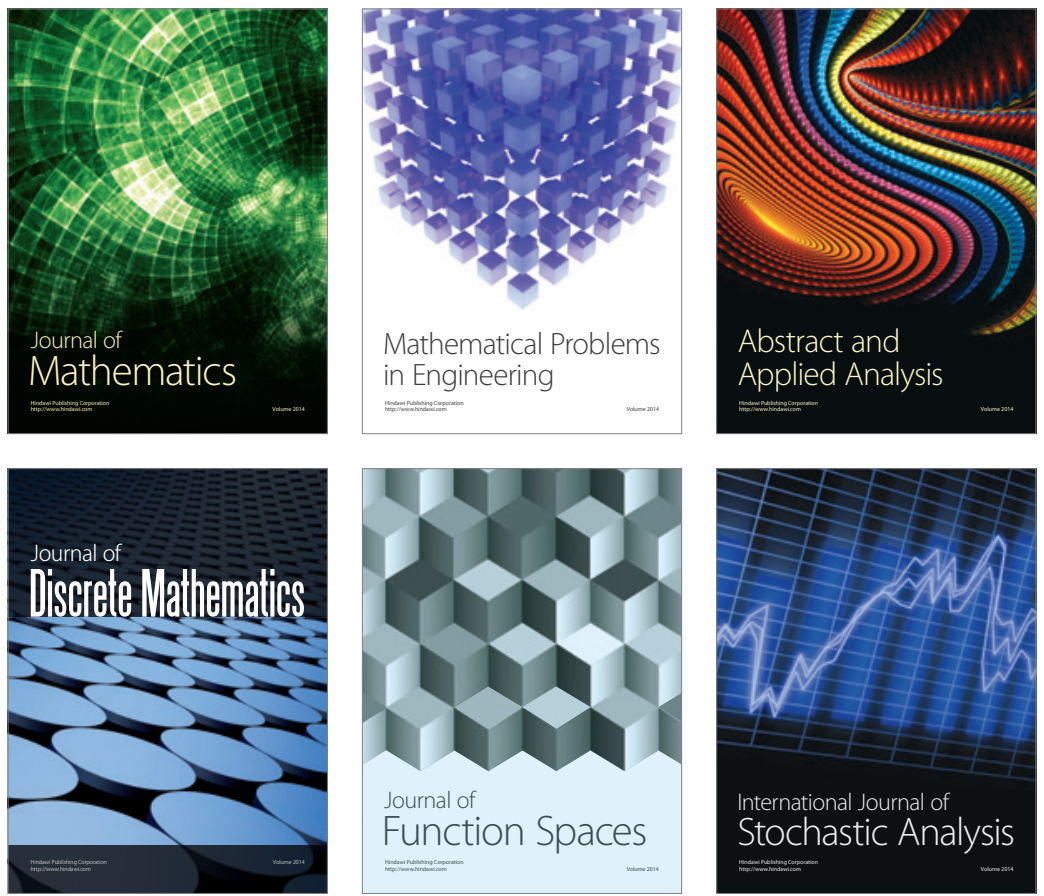

Journal of

Function Spaces

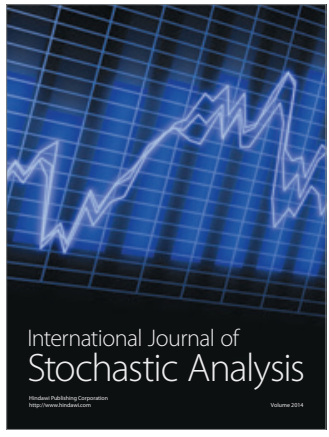

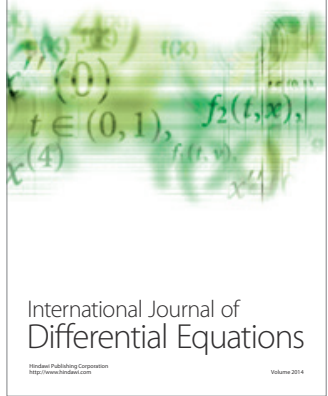
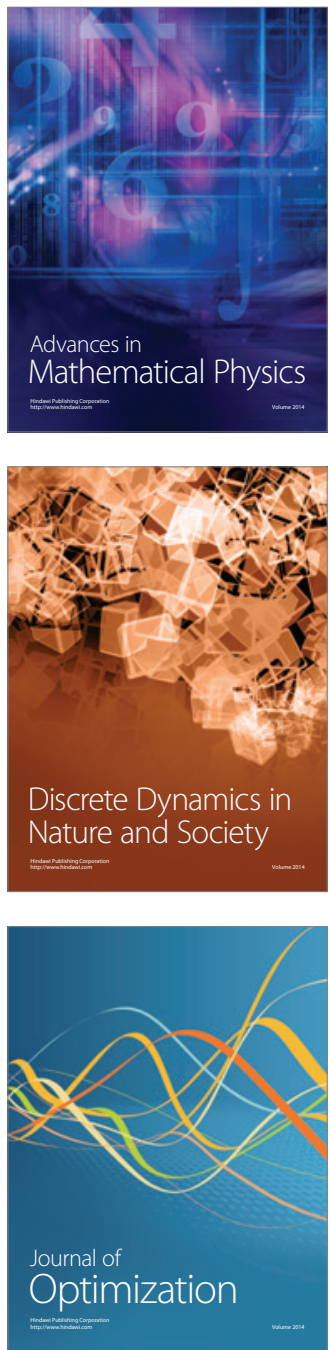